\title{
FLORA DO ESTRATO HERBÁCEO DA RESTINGA DA PRAIA DO CAÚRA, SÃO JOSÉ DE RIBAMAR, MARANHÃO
}

\author{
Aryana Vasque Frota Guterres ${ }^{1 *(D)}$, Ingrid Fabiana Fonseca Amorim ${ }^{2}$ (D); Antonio Fernando Costa da
} Silva ${ }^{(D)}$ Eduardo Bezerra de Almeida $\mathrm{Jr}^{3}$ (iD).

${ }^{1}$ Programa de Pós-graduação em Botânica, Universidade Estadual de Feira de Santana, Departamento de Ciências Biológicas, Av. Transnordestina s/n, Novo Horizonte, Feira de Santana, Bahia, Brasil.

${ }^{2}$ Programa de Pós-Graduação em Biodiversidade e Biotecnologia da Amazônia Legal (BIONORTE), Universidade Federal do Maranhão, Cidade Universitária Dom Delgado, Av. dos Portugueses, 1966. São Luís, MA, Brasil.

${ }^{3}$ Departamento de Biologia, Universidade Federal do Maranhão, Cidade Universitária Dom Delgado, Av. dos Portugueses, 1966. São Luís, MA, Brasil

*Autor correspondente: ana.vasque41@gmail.com

\section{RESUMO}

Este estudo teve por objetivo registrar a flora do componente herbáceo da restinga da Praia do Caúra, apontando a riqueza, formas de vida e trazendo contribuições para o conhecimento florístico do litoral maranhense. O estudo foi desenvolvido na restinga da Praia do Caúra $\left(02^{\circ} 33\right.$ '14,20"S, 4402'13,99'W), São José de Ribamar. Foram realizadas coletas mensais, do estrato herbáceo, entre 2015 a 2017, por meio de buscas exploratórias nos diferentes trechos da restinga. Foram identificadas 85 espécies, 68 gêneros e 28 famílias. As famílias mais representativas foram Fabaceae, com 16 espécies e Cyperaceae, com 12 espécies, seguido de Poaceae (9 spp), Asteraceae (6 spp), Euphorbiaceae e Passifloraceae, com 5 espécies, cada. A espécie Passiflora porophylla foi registrada pela primeira vez para o Maranhão. Foram identificados seis tipos de formas de vida (Caméfitos, Geófitos, Hemicriptófito, Holoparasita, Terófito e Trepadeira). Por fim, a riqueza registrada na área reforça a necessidade de manutenção da vegetação de restinga diante da perda da flora que vem acontecendo no local.

Palavras-chave: Riqueza de espécies, Formas de vida, Costa maranhense, Nordeste.

\section{FLORA OF HERBACEOUS STRATA IN RESTINGA OF THE CAÚRA BEACH, SÃO JOSÉ DE RIBAMAR, MARANHÃO STATE}

\begin{abstract}
This paper aimed to record the flora of the herbaceous component of restinga of Caúra beach, pointing out the richness, life-forms, and bringing contributions to the floristic knowledge of the coast of Maranhão state. The study was carried out in the restinga of Caúra beach $\left(02^{\circ} 33^{\prime} 14.20\right.$ "S, $44^{\circ} 02^{\prime} 13.99^{\prime \prime} W$ ), in the municipality of São José de Ribamar. Monthly collections of herbaceous strata were carried out between 2015 to 2017, through exploratory walks in the different stretches of the restinga. In the study area, 85 species, 68 genera and 28 families were identified. The most representative families were Fabaceae, with 16 species, and Cyperaceae, with 12 species, Poaceae (9 spp), Asteraceae (6 spp), Euphorbiaceae, and Passifloraceae, with 5 species, each. Passiflora porophylla was registered for the first time in Maranhão state. There were identified six types of life forms (chamaephytes, geophytes, Hemicryptophyte, Holoparasite, Terophyte and Vine. Therefore, the high number of species recorded in the area reinforces the need to maintain restinga vegetation because of the loss of flora that has been happening in the area.

Key-Word: Species richness, Life-forms, Coast Maranhão, Northeast.
\end{abstract}

\section{INTRODUÇÃO}

As restingas são caracterizadas por serem um complexo de formações vegetais constituídas por espécies arbóreas, arbustivas e herbáceas (SantosFilho \& Zickel, 2013), que se desenvolvem sob condições extremas como alta luminosidade, solo arenoso com altas taxas de salinidade, escassez de nutrientes e déficit hídrico (Scarano et al., 2001).

O componente herbáceo é uma das principais formações vegetais das áreas de restinga, sendo caracterizada por seus estolões ou rizomas que se desenvolvem sob o solo, exercendo um papel importante na fixação da areia, com serviços 
ecossistêmicos importantes no processo de estabilização das dunas (Barcellos Falkenberg, 1999; Cordazzo et al., 2006; Araujo et al., 2016). Além disso, compõem o grupo de espécies pioneiras que contribuem para a regeneração do solo e no processo de sucessão vegetal (Amorim et al., 2016; Santos et al., 2019), sendo consideradas como um importante incremento para a riqueza da área.

Apesar de serem caracterizadas como Áreas de Preservação Permanente, as restingas sempre estão sujeitas a fortes influências antrópicas, que envolvem queimadas intencionais, construções de moradias e inclusão de espécies exóticas, além da deposição de resíduos sólidos (Rocha et al., 2004). Como consequência, percebe-se o aumento do processo de descaracterização florística dessas áreas (Rocha \& Bergallo, 1997), dificultando que a flora da restinga possa ser conservada diante da quantidade de espécies ruderais que estão se desenvolvendo nesses ambientes. Por isso torna-se evidente a necessidade de estudos florísticos em áreas de restingas, diante das ações antrópicas que tem intensificado a perda da diversidade vegetal encontrada nesses ambientes naturais (Defeo et al., 2009).

Considerando a costa do Maranhão, alguns autores têm contribuído para ampliar os registros sobre a flora do Estado, possibilitando conhecer a riqueza de espécies que compõem o ecossistema de dunas e restinga. Entre os trabalhos desenvolvidos, destacam-se Silva et al. (2016), nas dunas de São Marcos, em São Luís; Serra et al. (2016), na restinga da Merck, em São José de Ribamar; Amorim et al. (2016) nas dunas de Araçagi, entre os municípios de São José de Ribamar e Paço do Lumiar; Almeida Jr. et al. (2018) nas áreas de restinga das Reentrâncias Maranhenses e Lima \& Almeida Jr. (2018) na restinga de Panaquatira, em São José de Ribamar.

Diante dos esforços realizados para registrar as espécies da flora, novos estudos florísticos se mostram necessários para mais contribuições sobre o conhecimento das espécies das restingas do nordeste brasileiro. Nesse sentido, o presente estudo teve como objetivo inventariar a flora do componente herbáceo da Praia do Caúra, destacando a riqueza de espécies e as formas de vida.

\section{MATERIAL E MÉTODOS}

\section{Área de estudo}

O estudo foi desenvolvido na restinga da Praia do Caúra $\left(02^{\circ} 33^{\prime} 14,20^{\prime \prime} \mathrm{S}, 44^{\circ} 02^{\prime} 13,99^{\prime \prime} \mathrm{W}\right)$, região banhada pela baía de São José, localiza-se no município de São José de Ribamar (Figura 1), com extensão de $1 \mathrm{~km}$, aproximadamente. A praia é separada por um canal da zona urbana do município (Figuras 2A a 2F) e no seu aspecto geral apresenta uma superfície plana, sem a ocorrência de depósitos arenosos elevados, o que explica o fato da área apresentar o tipo de dunas primárias (permanentes ou móveis). A área apresenta um período seco entre os meses de agosto a dezembro e períodos chuvosos iniciando em janeiro e segue até junho. O clima foi caracterizado, de acordo com a classificação de Koppen (Alvares et al., 2013), do tipo Aw (tropical) com precipitação média em torno de $2000 \mathrm{~mm} / \mathrm{ano}$ e com temperatura média de $28^{\circ} \mathrm{C}$, com mínima de $25^{\circ} \mathrm{C}$ e a máxima de $33^{\circ} \mathrm{C}$ (INMET, 2020).

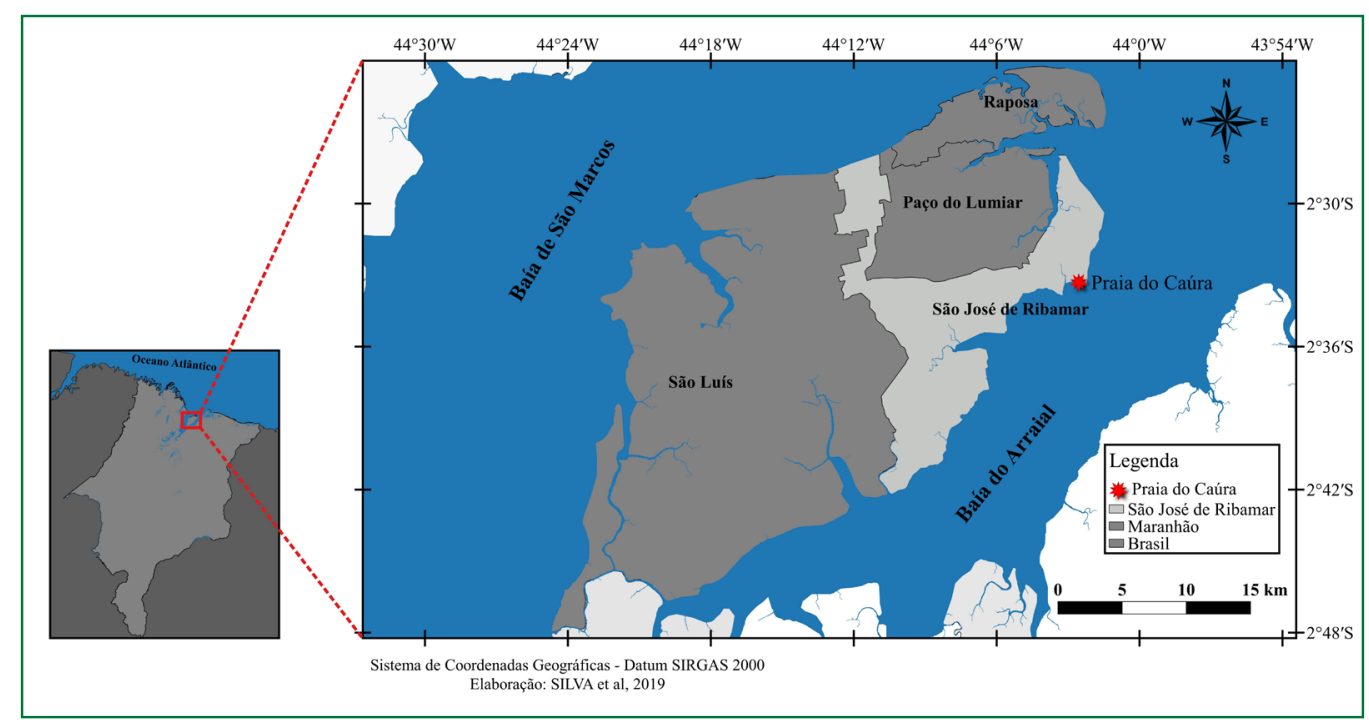

Figura 1. Mapa indicando a localização da Praia do Caúra, situada no município de São José de Ribamar, na porção nordeste da Ilha do Maranhão, Maranhão. 
Foram evidenciados para a área os campos abertos e fechados, e em alguns trechos foram observadas inundações permanentes, além das áreas manguezais que estão sujeitas a influência das marés. Foi registrado, no pós-praia, a ocorrência de falésia em processo de degradação com grande extensão. Ao longo da maior parte do trajeto da praia, onde foi realizado o levantamento florístico, foram observados fortes indícios de ocupação humana, com presença de construções de casas e demarcações de terrenos, o que evidencia uma alta especulação imobiliária.

\section{Coleta florística do estrato herbáceo}

As coletas das plantas ocorreram mensalmente, entre agosto de 2015 a maio de 2017, através de caminhadas exploratórias ao longo de toda a área. Foram coletadas todas as ervas e subarbustos que estivessem em estado fértil (com flor ou fruto); além disso, foram anotadas em caderneta de campo características importantes para o diagnóstico das espécies, como porte, odor, cor, presença de látex entre outras.

O processamento das amostras botânicas foi realizado de acordo com Peixoto \& Maia (2013). Todo material foi identificado no Laboratório de Estudos Botânicos (LEB) na Universidade Federal do Maranhão com apoio de bibliografias específicas e chaves de identificação (Filardi et al., 2007; Trevisan et al., 2008; Mondin et al., 2011; Pereira \& Kinoshita, 2013; Roque et al., 2017), além de comparação com material já identificado no acervo do Herbário MAR (Universidade Federal do Maranhão), onde os espécimes identificados foram depositados. A organização das famílias seguiu a proposta de classificação do APG IV (2016) e para a classificação das formas de vida das espécies foi seguida a proposta de Raunkiaer (1934), com alterações por Martins \& Batalha (2011).

\section{RESULTADOS}

Foram identificadas 85 espécies herbáceas, distribuídas em 68 gêneros e 28 famílias (Tabela 1). As famílias mais representativas foram Fabaceae, com 16 espécies e Cyperaceae com 12 espécies, seguido de Poaceae (9 spp), Asteraceae (6 spp), Euphorbiaceae e Passifloraceae com 5 espécies, cada. As espécies mais observadas na área foram Cyperus ligularis, Euphorbia hyssopifolia, Chamaecrista ramosa, Centrosema brasilianum e Eleocharis obtusetrigona. Além disso, 77 espécies registradas na área foram classificadas como nativas, desenvolvendo-se nesses ambientes costeiros.

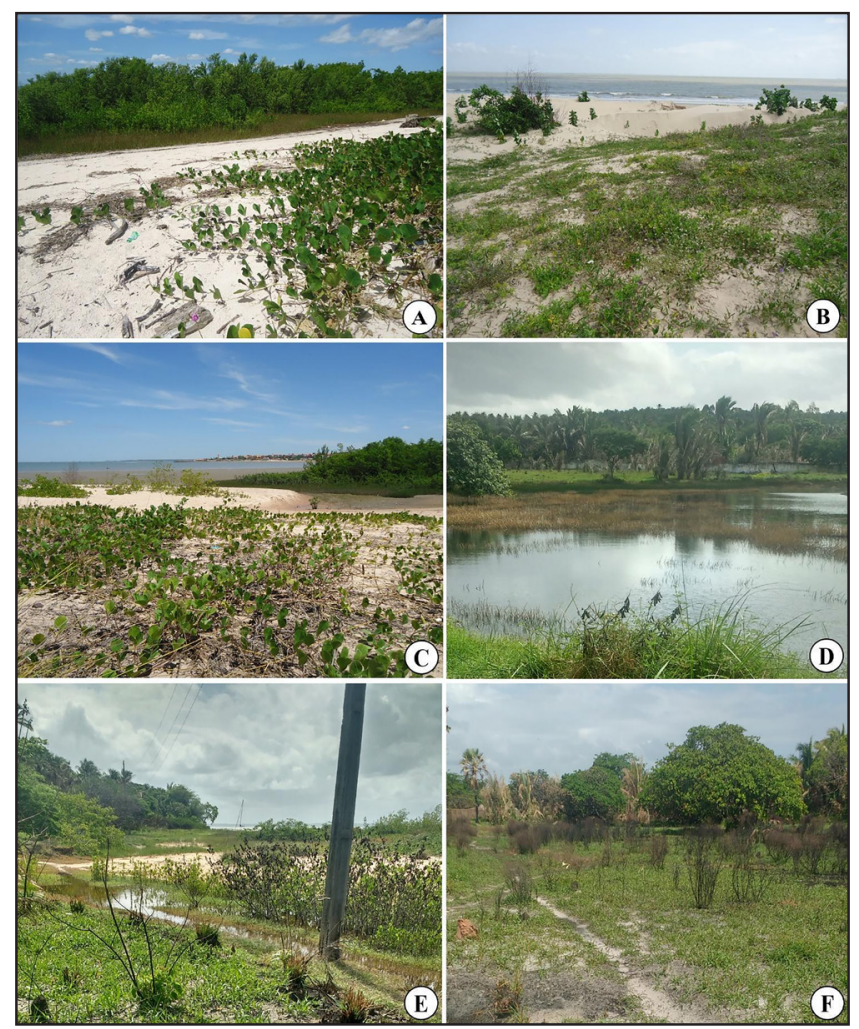

Figura 2. (A-F) Imagens da vegetação de restinga com destaque para o mosaico de fitofisionomias registradas na Praia do Caúra, São José de Ribamar, Maranhão. 
Tabela 1. Lista de espécies do estrato herbáceo identificadas na restinga da Praia do Caúra, São José de Ribamar, Maranhão e suas respectivas famílias e formas de vida. Legenda: Nat = Nativa; Natz = Naturalizada.

\begin{tabular}{|c|c|c|c|}
\hline Famílias/ Espécies & Origem & Formas de vida & Nome e número de coletor \\
\hline \multicolumn{4}{|l|}{ AIZOACEAE } \\
\hline Sesuvium portulacastrum (L.) L. & Natz & Caméfito & Guterres, A.V.F. 10776 \\
\hline \multicolumn{4}{|l|}{ AMARANTHACEAE } \\
\hline Alternanthera tenella Colla & Nat & Terófito & Guterres, A.V.F. 10861 \\
\hline Blutaparon portulacoides (A.St.-Hil.) Mears & Nat & Caméfito & Amorim, I.F.F. 10455 \\
\hline \multicolumn{4}{|l|}{ AMARYLLIDACEAE } \\
\hline Griffinia sp. & Nat & Geófito & Guterres, A.V.F. 11475 \\
\hline Hippeastrum elegans (Spreng.) H.E.Moore & Nat & Geófito & Guterres, A.V.F. 485 \\
\hline \multicolumn{4}{|l|}{ ASTERACEAE } \\
\hline Bidens riparia Kunth & Nat & Terófito & Guterres, A.V.F. 11375 \\
\hline Eclipta prostrata (L.) L. & Nat & Caméfito & Guterres, A.V.F. 11374 \\
\hline Elephantopus mollis Kunth & Nat & Terófito & Guterres, A.V.F. 11360 \\
\hline Eleutheranthera ruderalis (Sw) Sch.Bip. & Natz & Caméfito & Guterres, A.V.F. 11469 \\
\hline Emilia sonchifolia (L.) DC. & Natz & Terófito & Guterres, A.V.F. 11369 \\
\hline Mikania cordifolia (L.f.) Willd. & Nat & Trepadeira & Guterres, A.V.F. 10761 \\
\hline Tridax procumbens L. & Natz & Terófito & Guterres, A.V.F. 10875 \\
\hline \multicolumn{4}{|l|}{ BORAGINACEAE } \\
\hline $\begin{array}{l}\text { Euploca polyphylla (Lehm.) J.I.M.Melo \& } \\
\text { Semir }\end{array}$ & Nat & Hemicriptófito & Amorim, I. F. F. 9370 \\
\hline \multicolumn{4}{|l|}{ COMMELINACEAE } \\
\hline Commelina erecta L. & Nat & Terófito & Guterres, A.V.F. 10855 \\
\hline \multicolumn{4}{|l|}{ CONVOLVULACEAE } \\
\hline Ipomoea imperati (Vahl) Griseb. & Nat & Caméfito & Guterres, A.V.F. 10881 \\
\hline Ipomoea mauritiana Jacq. & Nat & Caméfito & Guterres, A.V.F. 10915 \\
\hline Ipomoea pes-caprae (L.) R.Br. & Nat & Caméfito & Guterres, A.V.F. 11468 \\
\hline \multicolumn{4}{|l|}{ CUCURBITACEAE } \\
\hline Ceratosanthes palmata (L.) Urb. & Nat & Trepadeira & Guterres, A.V.F. 11456 \\
\hline \multicolumn{4}{|l|}{ CYPERACEAE } \\
\hline Bulbostylis capillaris (L.) C.B.Clarke & Nat & Terófito & Guterres, A.V.F. 10857 \\
\hline Cyperus aggregatus (Willd.) Endl. & Nat & Terófito & Amorim, I. F. F. 418 \\
\hline Cyperus haspan L. & Nat & Terófito & Guterres, A.V.F. 10763 \\
\hline Cyperus ligularis L. & Nat & Terófito & Guterres, A.V.F. 10852 \\
\hline Cyperus polystachyos Rottb. & Nat & Terófito & Guterres, A.V.F. 10880 \\
\hline Cyperus sesquiflorus (Torr.) Mattf. \& Kük. & Nat & Terófito & Guterres, A.V.F. 10867 \\
\hline Cyperus surinamensis Rottb. & Nat & Terófito & Guterres, A.V.F. 10766 \\
\hline Eleocharis geniculata (L.) Roem. \& Schult. & Nat & Terófito & Guterres, A.V.F. 10887 \\
\hline $\begin{array}{l}\text { Eleocharis obtusetrigona (Lindl. \& Nees) } \\
\text { Steud. }\end{array}$ & Nat & Terófito & Guterres, A.V.F. 10778 \\
\hline Fimbristylis cymosa $\mathrm{R} . \mathrm{Br}$. & Nat & Hemicriptófito & Guterres, A.V.F. 10755 \\
\hline
\end{tabular}




\begin{tabular}{|c|c|c|c|}
\hline Famílias/ Espécies & Origem & Formas de vida & Nome e número de coletor \\
\hline Fuirena umbellata Rottb. & Nat & Terófito & Guterres, A.V.F. 10851 \\
\hline Rhynchospora holoschoenoides (Rich.) Herter & Nat & Terófito & Guterres, A.V.F. 10767 \\
\hline \multicolumn{4}{|l|}{ EUPHORBIACEAE } \\
\hline Cnidoscolus urens (L.) Arthur & Nat & Caméfito & Guterres, A.V.F. 11460 \\
\hline Croton hirtus L’Hér. & Nat & Terófito & Guterres, A.V.F. 10781 \\
\hline Euphorbia hirta L. & Nat & Hemicriptófito & Guterres, A.V.F. 10752 \\
\hline Euphorbia hyssopifolia L. & Nat & Hemicriptófito & Guterres, A.V.F. 300 \\
\hline Microstachys corniculata (Vahl) Griseb. & Nat & Terófito & Guterres, A.V.F. 10768 \\
\hline \multicolumn{4}{|l|}{ FABACEAE } \\
\hline $\begin{array}{l}\text { Ctenodon brevipes (Benth.) D.B.O.S. Cardoso, } \\
\text { P.L.R. Moraes \& H.C.Lima }\end{array}$ & Nat & Caméfito & Guterres, A.V.F. 10892 \\
\hline Canavalia rosea (Sw.) DC. & Nat & Trepadeira & Guterres, A.V.F. 10872 \\
\hline Centrosema brasilianum (L.) Benth. & Nat & Trepadeira & Amorim, I. F. F. 9358 \\
\hline Chamaecrista diphylla (L.) Greene & Nat & Hemicriptófito & Guterres, A.V.F. 10902 \\
\hline $\begin{array}{l}\text { Chamaecrista hispidula (Vahl) H.S.Irwin \& } \\
\text { Barneby }\end{array}$ & Nat & Hemicriptófito & Guterres, A.V.F. 10906 \\
\hline Crotalaria retusa L. & Natz & Terófito & Guterres, A.V.F. 10756 \\
\hline Desmodium barbatum (L.) Benth. & Nat & Terófito & Amorim, I. F. F. 9373 \\
\hline Galactia striata (Jacq.) Urb. & Nat & Trepadeira & Amorim, I. F. F. 9357 \\
\hline Macroptilium gracile (Poepp. ex Benth.) Urb. & Nat & Terófito & Guterres, A.V.F. 10897 \\
\hline Mimosa candollei R. Grether & Nat & Caméfito & Guterres, A.V.F. 10905 \\
\hline Mimosa pudica L. & Nat & Caméfito & Guterres, A.V.F. 11467 \\
\hline Neptunia plena (L.) Benth. & Nat & Caméfito & Guterres, A.V.F. 356 \\
\hline Rhynchosia minima (L.) DC. & Nat & Hemicriptófito & Guterres, A.V.F. 10753 \\
\hline Stylosanthes angustifolia Vogel & Nat & Caméfito & Guterres, A.V.F. 10882 \\
\hline Zornia guanipensis Pittier & Nat & Caméfito & Guterres, A.V.F. 10901 \\
\hline Zornia reticulata $\mathrm{Sm}$. & Nat & Caméfito & Guterres, A.V.F. 10750 \\
\hline \multicolumn{4}{|l|}{ GENTIANACEAE } \\
\hline Schultesia guianensis (Aubl.) Malme & Nat & Terófito & Guterres, A.V.F. 10876 \\
\hline \multicolumn{4}{|l|}{ HELICONIACEAE } \\
\hline Heliconia psittacorum L.f. & Nat & Terófito & Guterres, A.V.F. 10885 \\
\hline \multicolumn{4}{|l|}{ HYDROLEACEAE } \\
\hline Hydrolea spinosa L. & Nat & Terófito & Guterres, A.V.F. 11473 \\
\hline \multicolumn{4}{|l|}{ LAMIACEAE } \\
\hline Amasonia arborea Kunth & Nat & Terófito & Guterres, A.V.F. 11367 \\
\hline Marsypianthes chamaedrys (Vahl) Kuntze & Nat & Hemicriptófito & Amorim, I. F. F. 9338 \\
\hline \multicolumn{4}{|l|}{ LAURACEAE } \\
\hline Cassytha filiformis L. & Nat & Holoparasita & Guterres, A.V.F. 10863 \\
\hline \multicolumn{4}{|l|}{ LOGANIACEAE } \\
\hline Spigelia anthelmia $\mathrm{L}$. & Nat & Terófito & Guterres, A.V.F. 10870 \\
\hline \multicolumn{4}{|l|}{ MELASTOMATACEAE } \\
\hline Pterolepis glomerata (Rottb.) Miq. & Nat & Terófito & Guterres, A.V.F. 11466 \\
\hline
\end{tabular}




\begin{tabular}{|c|c|c|c|}
\hline Famílias/ Espécies & Origem & Formas de vida & Nome e número de coletor \\
\hline \multicolumn{4}{|l|}{ MOLLUGINACEAE } \\
\hline Mollugo verticillata $\mathrm{L}$. & Nat & Terófito & Guterres, A.V.F. 10871 \\
\hline \multicolumn{4}{|l|}{ ONAGRACEAE } \\
\hline Ludwigia leptocarpa (Nutt.) H.Hara & Nat & Caméfito & Amorim, I. F. F. 9354 \\
\hline \multicolumn{4}{|l|}{ OROBANCHACEAE } \\
\hline Buchnera palustris (Aubl.) Spreng. & Nat & Caméfito & Guterres, A.V.F. 10865 \\
\hline \multicolumn{4}{|l|}{ PASSIFLORACEAE } \\
\hline Passiflora foetida $\mathrm{L}$. & Nat & Trepadeira & Guterres, A.V.F. 10784 \\
\hline Passiflora porophylla Vell. & Nat & Trepadeira & Guterres, A.V.F. 10916 \\
\hline Piriqueta duarteana (Cambess.) Urb. & Nat & Caméfito & Guterres, A.V.F. 355 \\
\hline Turnera pumilea $\mathrm{L}$. & Nat & Caméfito & Guterres, A.V.F. 10758 \\
\hline Turnera subulata $\mathrm{Sm}$. & Nat & Caméfito & Guterres, A.V.F. 10873 \\
\hline \multicolumn{4}{|l|}{ PLANTAGINACEAE } \\
\hline Bacopa sp. & Nat & Terófito & Guterres, A.V.F. 11376 \\
\hline \multicolumn{4}{|l|}{ POACEAE } \\
\hline Andropogon leucostachyus Kunth & Nat & Terófito & Guterres, A.V.F. 11471 \\
\hline Cenchrus echinatus L. & Nat & Terófito & Guterres, A.V.F. 10908 \\
\hline Echinochloa colona $($ L.) Link & Natz & Terófito & Guterres, A.V.F. 11362 \\
\hline Digitaria horizontalis Willd. & Natz & Terófito & Guterres, A.V.F. 11472 \\
\hline $\begin{array}{l}\text { Megathyrsus maximus (Jacq.) B.K.Simon \& } \\
\text { S.W.L. Jacobs }\end{array}$ & Natz & Terófito & Guterres, A.V.F. 10891 \\
\hline Paspalum maritimum Trin. & Nat & Terófito & Guterres, A.V.F. 10772 \\
\hline Sporobolus indicus (L.) R.Br. & Nat & Terófito & Guterres, A.V.F. 10770 \\
\hline Sporobolus virginicus (L.) Kunth & Nat & Terófito & Guterres, A.V.F. 10777 \\
\hline Streptostachys asperifolia Desv. & Nat & Terófito & Guterres, A.V.F. 10774 \\
\hline \multicolumn{4}{|l|}{ POLYGALACEAE } \\
\hline $\begin{array}{l}\text { Asemeia martiana (A.W.Benn.) J.F.B.Pastore \& } \\
\text { J.R.Abbott }\end{array}$ & Nat & Caméfito & Guterres, A.V.F. 11464 \\
\hline Polygala sp. & Nat & Caméfito & Guterres, A.V.F. 378 \\
\hline \multicolumn{4}{|l|}{ RUBIACEAE } \\
\hline Borreria latifolia (Aubl.) K.Schum. & Nat & Caméfito & Guterres, A.V.F. 384 \\
\hline Borreria verticillata (L.) G.Mey. & Nat & Caméfito & Guterres, A.V.F. 10874 \\
\hline $\begin{array}{l}\text { Mitracarpus strigosus (Thunb.) P.L.R.Moraes, } \\
\text { De Smedt \& Hjertson }\end{array}$ & Nat & Caméfito & Guterres, A.V.F. 224 \\
\hline \multicolumn{4}{|l|}{ VIOLACEAE } \\
\hline Pombalia calceolaria (L.) Paula-Souza & Nat & Caméfito & Guterres, A.V.F. 11455 \\
\hline \multicolumn{4}{|l|}{ XYRIDACEAE } \\
\hline Xyris anceps Lam. & Nat & Terófito & Amorim, I. F. F.10420 \\
\hline Xyris macrocephala Vahl & Nat & Terófito & Guterres, A.V.F. 10860 \\
\hline
\end{tabular}


A partir deste inventário foi verificado que a espécie Passiflora porophylla está sendo registrada pela primeira vez para o Maranhão. Essa espécie é caracterizada como uma trepadeira nativa e ocorre em toda a região sudeste e sul do país. No Nordeste, só tinha registro para a Bahia (Flora do Brasil, 2020).

Em relação às formas de vida, foram identificados seis tipos: Caméfitos, Geófitos, Hemicriptófito,
Holoparasita, Terófito e Trepadeira (Figura 3). $\mathrm{Na}$ área, as formas de vida predominantes foram Terófitos, com 41 espécies, seguido de Caméfito, com 26 e Hemicriptófito, com oito espécies. A partir da listagem florística foi elaborada uma prancha fotográfica com as espécies mais comuns coletadas na área de estudo (Figura 4).

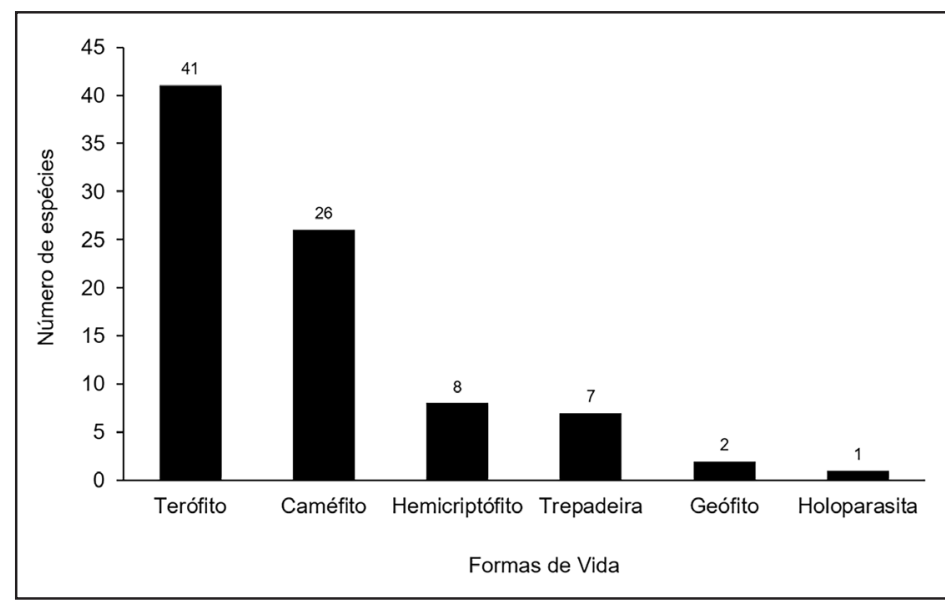

Figura 3. Distribuição das espécies inventariadas de acordo com as formas de vida na restinga da Praia do Caúra, São José de Ribamar, Maranhão.
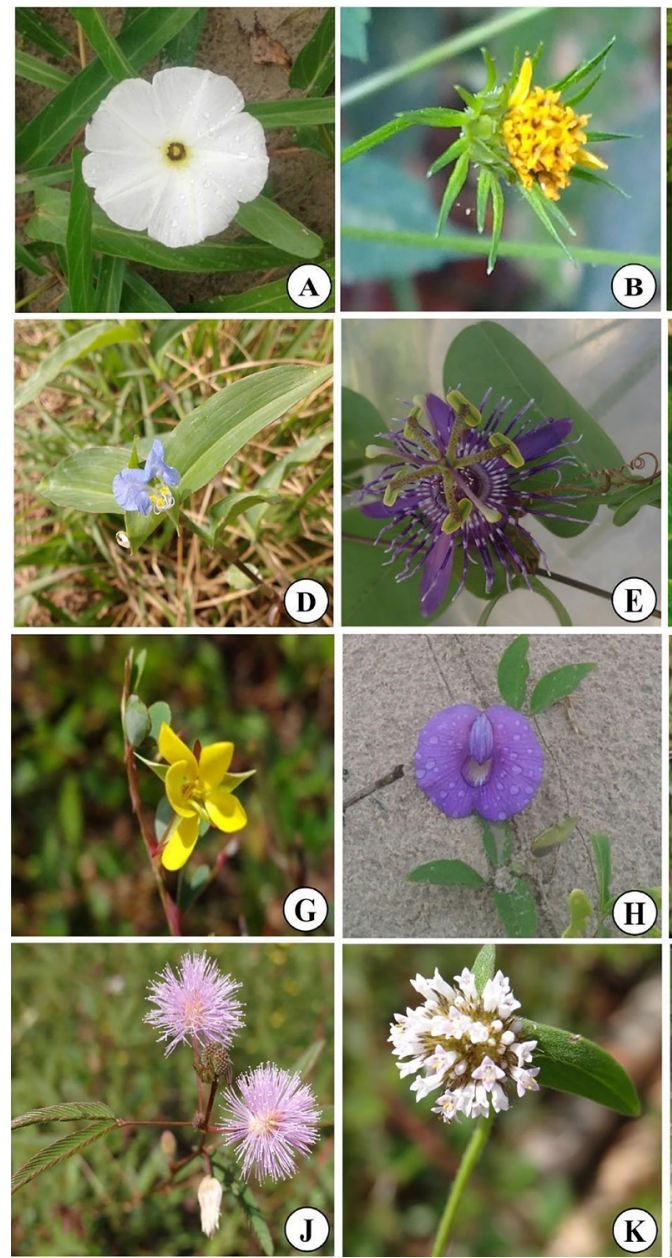
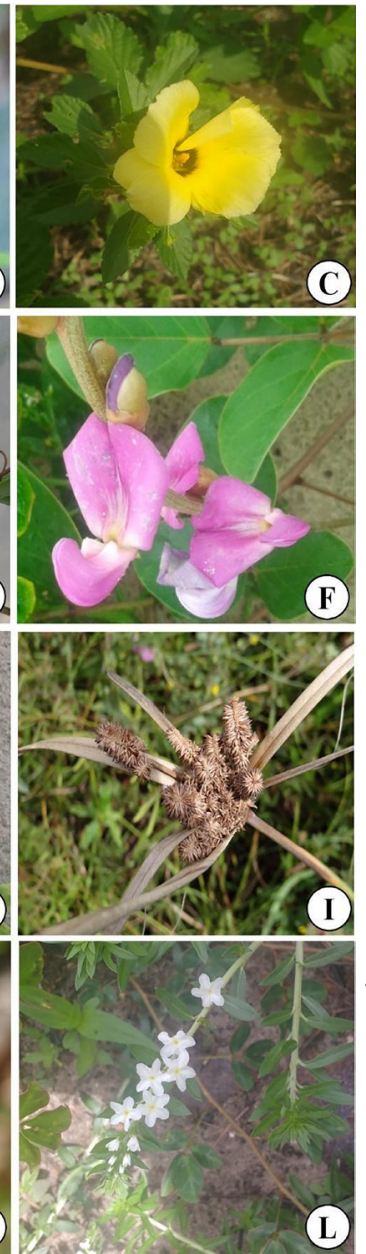

(L)
Figura 4. A-L: Espécies coletadas na área de estudo. A-Ipomoea imperati (Vahl) Griseb.; B-Bidens riparia Kunth.; C- Turnera subulata Sm.; DCommelina erecta L.; E- Passiflora foetida L.; F- Canavalia rosea (Sw.) DC.; G- Chamaecrista diphylla (L.) Greene.; H- Centrosema brasilianum (L.) Benth.; I- Cyperus ligularis L.; J- Mimosa pudica L.; K Mitracarpus strigosus (Thunb.).; L- Euploca polyphylla (Lehm.) J.I.M.Melo \& Semir. 
A heterogeneidade observada nas fisionomias de campo no presente estudo influenciou diretamente na composição de espécies, visto que nos fragmentos inundáveis houve a predominância das espécies Eleocharis geniculata, Eleocharis obtusetrigona, Fimbristylis cymosa, Andropogon leucostachyus e Heliconia psittacorum, que são associadas a ambientes úmidos. Em relação aos trechos do campo em que não apresentava inundações, as espécies Sesuvium portulacastrum e Alternanthera tenella e Turnera pumilea foram mais frequentes.

\section{DISCUSSÃO}

Na restinga da Praia do Caúra, o estrato herbáceo contribui de forma expressiva para a riqueza da área. A predominância desse estrado também foi evidenciada em outras áreas de restinga do Maranhão (Amorim et al., 2016; Araujo et al., 2016; Santos et al., 2019) por ser importante, principalmente, na fixação das dunas (Araujo et al., 2016). Apesar dos fatores adversos comuns às restingas, o componente herbáceo consegue se estabelecer, juntamente com a vegetação halófila-psamófila (Arruda et al., 2009) permitindo que outras espécies mais exigentes possam se desenvolver na área (Santos et al., 2019).

As famílias Fabaceae, Cyperaceae e Poaceae, em termos de riqueza específica, estão entre as mais registradas em áreas de restinga (Macedo et al., 2007; Almeida Jr. \& Zickel, 2009; Silva et al., 2016). O sucesso destas famílias pode estar associado ao fato das espécies apresentarem estratégias xeromórficas de escape para fatores abióticos, como a presença de tricomas, espessamento da cutícula e redução da lâmina foliar (Pereira et al., 2003; Ferreira et al., 2015), permitindo assim que essas plantas possam se desenvolver nesses ambientes.

Em relação ao novo registro de ocorrência de Passiflora porophylla, cabe destacar a importância dos estudos florísticos para a ampliação do conhecimento da riqueza biológica e da distribuição geográfica das espécies que se desenvolvem nas restingas do Maranhão. Esse novo registro pode colaborar com a compreensão da abrangência geográfica desse grupo taxonômico, possibilitando que outros pesquisadores conheçam as variações morfológicas regionais das plantas (Dias \& Almeida Jr., 2017).

Quanto às formas de vida, o destaque dos terófitos e dos caméfitos na Praia do Caúra evidencia o sucesso desse grupo que apresenta mecanismos fisiológicos e morfológicos que possibilitam seu desenvolvimento em áreas de restinga (Martins \& Batalha, 2011). Mesmo com a baixa oferta de recursos para sua sobrevivência, os terófitos conseguem completar seu ciclo de vida dentro da estação favorável (Martins \& Batalha, 2011) e suas sementes conseguem resistir aos fatores limitantes do ambiente, como exemplo: Cyperus ligularis, Commelina erecta, Alternanthera tenella e Crotalaria retusa.

A maior ocorrência de caméfitos também pode ser explicada pelos ajustes morfológicos que essas espécies possuem (Martins \& Batalha, 2011) para conseguir se desenvolver nas áreas de restinga, além de suportar ventos fortes, alta salinidade e influência das marés, como Turnera pumilea, Ipomoea pescaprae e Mimosa candollei.

A espécie Cassytha filiformis foi a única holoparasita encontrada na área. Essa planta merece especial atenção por apresentar características fisiológicas que extraem os recursos de suas hospedeiras para seu desenvolvimento (Nelson, 2008). Isso compromete a vegetação do estrato herbáceo porque a $C$. filiformis cresce sobre as plantas, cobrindo a vegetação (Gomes \& Guedes, 2014) com seus ramos de coloração verde-alaranjada, formando densos e extensos emaranhados.

Por ser próximo ao perímetro urbano, foram evidentes ao longo da vegetação de restinga da Praia de Caúra indícios de perturbações antrópicas e resíduos sólidos (material plástico, material de construção, entre outros) em grandes quantidades; além de trechos onde eram descartados lixo orgânico. Somado a isso, também foram registrados focos de queimadas (intencionais ou acidentais), afetando fortemente a vegetação ao longo do ano. Cabe ressaltar que devido ao grau de perturbação observado, somados aos fatores limitantes comuns as restingas (Araujo et al., 2016), a vegetação da área terá mais dificuldades para o seu reestabelecimento e/ou regeneração natural.

Apesar da riqueza apontada para a vegetação do estrato herbáceo da restinga da Praia do Caúra, esse valor deve ser analisado com cautela pois pode estar relacionado à antropização na área que contribui com a entrada de espécies ruderais. Isso ocasiona mais competição com as espécies nativas e podendo inibir ou dificultar a entrada e/ou estabelecimento de espécies típicas de restingas (Holl, 2002; Rocha et al., 2007).

\section{CONCLUSÃO}

Diante dos dados apresentados, pode-se concluir que a riqueza do estrato herbáceo quando comparada a outras áreas de dunas do litoral do 
Maranhão, reforça a necessidade de conservação da flora da restinga na Praia do Caúra, diante da acelerada perda de vegetação que vem acontecendo no local. Além disso, essas espécies contribuem para a estabilização do solo e são consideradas como pioneiras, gerando condições para o estabelecimento de espécies de diferentes portes.

Através desse estudo foi possível verificar que a antropização tem afetado as restingas, contribuindo para a descaracterização da composição florística das áreas litorâneas do Estado. Com isso, novos estudos sempre serão essenciais para acompanhar o registro das plantas e subsidiar futuras pesquisas, gerando dados para fins ecológicos, fisiológicos e taxonômicos.

\section{AGRADECIMENTOS}

Ao Conselho Nacional de Desenvolvimento Científico e Tecnológico (CNPq) pela bolsa PIBIC/ $\mathrm{CNPq} / \mathrm{UFMA}$ da primeira autora. A Fundação de Amparo à Pesquisa e ao Desenvolvimento Científico e Tecnológico do Maranhão (FAPEMA) pelo auxílio financeiro e bolsa produtividade do último autor. A Universidade Federal do Maranhão (UFMA) e ao Laboratório de Estudos Botânicos (LEB) pela estrutura física e recursos humanos.

\section{REFERÊNCIAS}

ALMEIDA JR., E.B., GUTERRES, A.V.F., AMORIM, G.S., ANJOS, J.S., COSTA, L.B.S., LIMA, G.P., AMORIM, I.F.F., DINIZ, M.R., DIAS, K.N.L. \& SILVA, A. N. F. 2018. Expedição botânica às reentrâncias maranhenses: contribuições para o conhecimento da flora do Estado. In: SANTOS-FILHO, F S. \& ALMEIDA JR., E. B. (Org.). Biodiversidade do Meio Norte do Brasil: conhecimentos ecológicos e aplicações. 1ed. Curitiba: CRV, p. 111-136.

ALMEIDA JR., E.B. \& ZICKEL, C.S. 2009. Fisionomia psamófila-reptante: riqueza e composição de espécies na praia da pipa, rio Grande do Norte, Brasil. Pesquisas, Botânica, 60 (1): 289-299.

ALVARES, C.A., STAPE, J.L., SENTELHAS, P.C., MORAES, G., LEONARDO, J., \& SPAROVEK, G. 2013. KOPPEN. W. Climate classification map for Brazil. Meteorol, 22: 711-718.

AMORIM, G.S., AMORIM, I.F.F. \& ALMEIDA JR., E.B. 2016. Flora de uma área de dunas antropizadas na praia de Araçagi, Maranhão. Revista Biociências, 22(2): 18-29.
APG IV. 2016. An update of the Angiosperm Phylogeny Group classification for the orders and families of flowering plants. Botanical Journal of the Linnean Society, 181: 1-20.

ARAUJO, A.C.M., SILVA, A.N.F. \& ALMEIDA JR., E.B. 2016. Caracterização estrutural e status de conservação do estrato herbáceo de dunas da Praia de São Marcos, Maranhão, Brasil. Acta Amazonica, 46 (3): 247-258.

ARRUDA, R.C.O., VIGLIO, N.S.F. \& BARROS, A.A.M. 2009. Anatomia foliar de halófitas e psamófilas reptantes ocorrentes na restinga de Ipitangas, Saquarema, Rio de Janeiro, Brasil. Rodriguésia, 60 (2): 333-352.

BARCELLOS FALKENBERG, D. 1999. Aspecto da flora e da vegetação secundária da Restinga de Santa Catarina, sul do Brasil. Insula Revista de Botânica, 28:1-30.

CORDAZZO, C.V., PAIVA, J.B. \& SEELIGER, U. 2006. Plantas de dunas da costa sudeste atlântica. Useb, Pelotas. 107 p.

DEFEO, O., MCLACHLAN, A., SCHOEMAN, D.S., SCHLACHER, T. A., DUGAN, J., JONES, A., LASTRA, M. \& SCAPINI, F. 2009. Threats to sandy beach ecosystems: A review. Estuarine, Coastal and Shelf Science, 81 (1): 1-12.

DIAS, K.N.L. \& ALMEIDA JR., E.B. 2017. Estudos taxonômicos para o Maranhão a partir do registro de nova ocorrência de Rottboellia cochinchinensis. Revista Trópica: Ciências Agrárias e Biológicas, 9(1): 98-103.

FERREIRA, C.S., CARMO, W.S., GRACIANORIBEIRO, D., OLIVEIRA, J.M.F., MELO, R.B. \& FRANCO, A.C. 2015. Anatomia da lâmina foliar de onze espécies lenhosas dominantes nas savanas de Roraima. Acta Amazonica, 45(4): 337-346.

FILARDI, F.L.R., GARCIA, F.C.P., DUTRA, V.F. \& SÃO-THIAGO, P.S. 2007. Papilionoideae (Leguminosae) do Parque Nacional da Serra da Canastra, Minas Gerais, Brasil. Hoehnea, 34 (3): 383-408.

FLORA DO BRASIL 2020. Jardim Botânico do Rio de Janeiro. Disponível em: < http://floradobrasil.jbrj. gov.br/ >. Acesso em: 27 Out. 2019.

GOMES, F.S. \& GUEDES, M.L.S. 2014. Flora vascular e formas de vida das formações de restinga do litoral norte da Bahia, Brasil. Acta Biológica Catarinense, 1(1): 22-43. 
HOLL, K.D. 2002. Effect of shrubs on tree seedling establisment in an abandoned tropical pasture. Journal of Ecology, 90(1): 179-187.

INMET. Instituto Nacional de Meteorologia. 2019. Disponível em: http://www.inmet.gov.br/ portal/. Acesso em: 04out. 2019.

LIMA, G.P. \& ALMEIDA JR., E.B. 2018. Diversidade e similaridade florística de uma Restinga ecotonal no Maranhão, Nordeste do Brasil. Interciencia, 43(4): 275-282.

MACEDO, T.S., FERNANDES, L.L., SILVA, D.F., VARJÃO, A.S., NEVES, A.S. \& PIGOZZO, C.M. 2007. Comparação florística entre um fragmento de mata atlântica e ambientes associados (restinga e manguezal) na cidade de Salvador, Bahia. Candombá - Revista Virtual, 3 (2): 138-148.

MARTINS, F.R. \& BATALHA, M.A. 2011. Formas de vida, espectro biológico de Raunkiaer e fisionomia da vegetação. In: FELFILI, J.M., EISENLOHR, P.V., MELO, M.M.R.F., MONDIN, C. A., CERVI, A. C. \& MOREIRA, G. R. P. 2011. Sinopse das espécies de Passiflora L. (Passifloraceae) do Rio Grande do Sul, Brasil. Brazilian Journal of Biosciences, 9(1): 3-27.

MONDIN, C. A., CERVI, A. C., \& MOREIRA, G. R. P. 2011. Sinopse das espécies de Passiflora L. (Passifloraceae) do Rio Grande do Sul, Brasil. Brazilian Journal of Biosciences, 9(1):3-27.

NELSON, S.C. 2008. Cassytha filiformis. Plant Disease, n. 42. 10p.

PEIXOTO, A.L. \& MAIA, L.C. 2013. Manual de Procedimentos para Herbários. INCT-Herbário virtual para a Flora e os Fungos. Recife: Editora Universitária UFPE. 97p.

PEREIRA, Z.V. \& KINOSHITA, L.S. 2013. Rubiaceae Juss. Do Parque Estadual das Várzeas do Rio Ivinhema, MS, Brasil. Hoehnea, 40(2): 205-251.

PEREIRA, Z.V., MEIRA, R.M.S.A. \& AZEVEDO, A.A. 2003. Morfoanatomia foliar de Palicourea longepedunculata Gardiner (Rubiaceae). Revista Árvore, 27(6): 759- 767.

RAUNKIAER, C. 1934. The Life Forms of Plants and Statistical Plant Geography. Oxford, Clarendon. $632 \mathrm{pp}$.

ROCHA, C.F.D. \& BERGALLO, H.G. 1997. Intercommunity variation in the distribution of abundance of dominant lizard species in resting habitats. Ciência e Cultura, 49(4): 269-274.
ROCHA, C.F.D., FREITAS, A.F.N., CARVALHO, L.C. \& ROCHA-PESSÔA, T.C. 2004.

Habitat disturbance in the Brazilian coastal sand dune vegetation and related richness and diversity of bromeliad species. Vidalia, 2 (2): 49-55.

ROCHA, C.F.D., BERGALLO, H.G., VAN SLUYS, M., ALVES, M.A.S. \& JAMEL, C.E. 2007. The remnants of restinga habitats in the brazilian Atlantic Forest of Rio de Janeiro state, Brazil: habitat loss and risk of disappearance. Brazilian Journal of Biology, 67(2): 263-273.

ROQUE, N., TELES, A.M. \& NAKAJIMA, J.N. 2017. A família Asteraceae no Brasil: classificação e diversidade. Salvador. EDUFBA. 260p.

SANTOS, C.R., AMORIM, I.F.F. \& ALMEIDA JR., E.B. 2019. Caracterização fitossociológica do componente halófilo-psamófilo em uma área de dunas, Maranhão, Brasil. Boletim do Laboratório de Hidrobiologia, 29(1):2-8.

SANTOS-FILHO, F.S. \& ZICKEL, C.S. 2013. Origem e estrutura da costa e vegetação de restinga: o caso do litoral do Piauí. In: SANTOS-FILHO, F.S., SOARES, A.F.C.L. \& ALMEIDA JR., E.B. (Org.). Biodiversidade do Piauí: pesquisa \& perspectivas Vol. 2. 1ed. Curitiba: CRV, p. 11-36.

SCARANO, F.R., DUARTE, H.M., RIBEIRO, K.T., RODRIGUES, P.J.F.P., BARCELLOS, E.M.B., FRANCO, A.C., BRULFERT, J., DELÉENS, E. \& LÜTTGE, U. 2001. Four sites with contrasting environmental stress in southeastern Brazil: relations of species, life form diversity, and geographical distribution to ecophysiological parameters. Botanical Journal of the Linnean Society, 136(4): 345-364.

SERRA, F.C.V., LIMA, P.B. \& ALMEIDA JR., E.B. 2016. Species richness in restinga vegetation on the eastern Maranhão State, Northeastern Brazil. Acta Amazonica, 46(3): 271-280.

SILVA, A.N.F., ARAUJO, A.N.M. \& ALMEIDA JR, E.B. 2016. Flora fanerogâmica das dunas da praia de São Marcos, Maranhão. In: SANTOS-FILHO, F.S \& ALMEIDA JR, E.B. (Eds.). Biodiversidade do Meio Norte do Brasil: Conhecimentos ecológicos e aplicações. Curitiba: CRV, p. 11-28.

TREVISAN, R., FERREIRA, P.M. \& BOLDRINI, I.I. 2008. A família Cyperaceae no Parque Estadual de Itapuã, Viamão, Rio Grande do Sul, Brasil. Revista Brasileira de Biociências, 6(3): 217-244. 